\title{
COVID-19: update in innovazione, ricerca e sviluppo
}

\author{
Francesco Burrai ${ }^{1}$, Luigi Apuzzo², Valentina Micheluzzi ${ }^{3}$ \\ ${ }^{1}$ Incaricato Progetti di Ricerca, SC Formazione, Ricerca e Cambiamento Organizzativo, ATS Sardegna, Sassari - Italia \\ ${ }^{2}$ Cardiologia d'emergenza con UTIC, AORN Caserta - Italia \\ ${ }^{3}$ Cardiochirurgia, AOU di Sassari, Sassari - Italia
}

\begin{abstract}
COVID-19: Innovation and research
Following the pandemic state, produced by the infection with the new coronavirus SARS-CoV-2, and the consequent state of health emergency, it is considered scientifically important to propose an update on ongoing clinical pharmacological trials, the most advanced international projects in the innovation sector and the most important statistical mathematical approaches to use a model for predictive purposes. In Italy there are several experimental molecules subjected to rigorous RCT studies approved by AIFA. In the Research \& Development sector, the CERN in Geneve with the "CERN against COVID-19 project" represents the most advanced innovation, while in predictive statistical techniques, the mathematical model with wavelet approach allows to predict variables such as the persistence of the virus or to calculate the probability of transmission, strategic information for health planning.
\end{abstract}

Keywords: COVID-19, Innovation, Pharma, Research, SARS-CoV-2

\section{Introduzione}

L'emergenza epidemiologica rappresentata dalla patologia "coronavirus disease 2019", abbreviata con l'acronimo "COVID-19", è causata dal SARS-CoV-2 virus, formalmente conosciuto come 2019-nCoV (1). L'11 marzo 2020 la WHO ha dichiarato COVID-19 una pandemia (2).

Al 2 maggio 2020, la WHO riporta 3.181 .642 casi confermati di COVID-19 e 224.301 deceduti a livello mondiale e 207.428 casi confermati con 28.236 deceduti in Italia al $1^{\circ}$ maggio (3).

La Global Surveillance for COVID-19 definisce il caso sospetto, il caso probabile e il caso confermato (4). Queste definizioni si basano su informazioni che subiscono un update periodico, mentre i vari paesi potrebbero dover produrre definizioni adattate in base alla situazione epidemiologica nazionale e in base ad altri fattori. La WHO incoraggia, dunque, i vari stati nazionali a pubblicare online le definizioni di

Received: May 11, 2020

Accepted: June 25, 2020

Published online: August 1, 2020

Indirizzo per la corrispondenza:

Francesco Burrai

SC Formazione, Ricerca e Cambiamento Organizzativo

ATS Sardegna

Sassari - Italia

francesco.burrai@atssardegna.it caso sospetto, caso probabile e caso confermato, nonché i loro aggiornamenti, i quali possono impattare sull'interpretazione dei dati di sorveglianza (4) e, dunque, anche sull'interpretazione dei dati provenienti dalla ricerca clinica.

In Italia, il Ministero della Salute, con la Circolare n. 6360 del 27/02/2020 “COVID-19" (5) adotta queste definizioni:

a) Caso sospetto. Una persona con infezione respiratoria acuta (insorgenza improvvisa di almeno uno tra i seguenti segni e sintomi: febbre, tosse e difficoltà respiratoria), che richiede o meno il ricovero ospedaliero e che soddisfi almeno uno dei seguenti criteri epidemiologici (riferiti al periodo di tempo dei 14 giorni prima della comparsa dei segni e dei sintomi):

- essere un contatto stretto di un caso confermato o probabile di COVID-19

oppure

- essere stato in zone con presunta trasmissione comunitaria (diffusa o locale).

b) Caso probabile. Un caso sospetto il cui risultato del test per SARS-CoV-2 è dubbio o inconcludente utilizzando protocolli specifici di Real Time PCR per SARS-CoV-2 presso i Laboratori di Riferimento Regionali individuati o è positivo utilizzando un test pan-coronavirus.

c) Caso confermato. Un caso con una conferma di laboratorio effettuata presso il laboratorio di riferimento dell'Istituto Superiore di Sanità per infezione da SARS-CoV-2, indipendentemente dai segni e dai sintomi clinici. 
La malattia COVID-19 non presenta ancora uno specifico trattamento terapeutico approvato dalla Food and Drug Administration né da altre autorità regolatorie internazionali. In questo momento, è presente un generale livello di incertezza delle evidenze disponibili (6), dunque sono estremamente necessari studi randomizzati controllati (RCT) rigorosi e ben disegnati per valutare l'efficacia e la sicurezza delle molecole sperimentali negli adulti ricoverati con diagnosi di COVID-19.

L'altro settore nella lotta contro il COVID-19 è quello della Ricerca \& Sviluppo, dove il progetto di collaborazione internazionale del CERN di Ginevra rappresenta, a oggi, il gold standard per l'innovazione.

Infine, proponiamo un interessante approccio matematico statistico, attraverso una modellistica matematica che permette di predire variabili, come, per esempio, la persistenza del virus, o di calcolare "la probabilità" di trasmissione dati, importante per la programmazione sanitaria.

Lo scopo dell'articolo, attraverso l'analisi della letteratura, è quello di mostrare in modo sistematico e completo tutti i principali trial farmacologici in corso, il principale modello matematico di stima di casi di COVID-19 e uno dei principali progetti internazionali di ricerca e innovazione in tema di COVID-19.

\section{Conseil Européen pour la Recherche Nucléaire: CERN against COVID-19}

La task force CERN Against COVID-19 nasce nel marzo 2020 per raccogliere e coordinare idee e contributi provenienti dalla comunità del CERN, formata da oltre 18.000 persone di tutto il mondo impegnate contro la pandemia di COVID-19. L'obiettivo dell'iniziativa CERN against COVID-19 è garantire un'azione efficace e ben coordinata, attingendo alle numerose competenze e tecnologie avanzate del CERN e lavorando a stretto contatto con esperti in sanità, lo sviluppo di farmaci, l'epidemiologia e la risposta alle emergenze, in modo da massimizzare l'impatto dei contributi dati.

Le aree d'azione sono: 1) Applicazioni mediche; 2) Informatica e analisi dei dati.

1) Applicazioni mediche. Molte iniziative sono in corso al CERN e nella più ampia comunità di fisica delle particelle per progettare e produrre forniture e attrezzature mediche. Questi progetti vanno dalla produzione di gel disinfettante allo sviluppo di ventilatori su vasta scala per I'uso ospedaliero. Altre iniziative al CERN sono l'utilizzo di strutture per officine e stampanti 3D per la produzione di dispositivi di protezione individuale, quali maschere, componenti per adattare maschere disponibili in commercio per uso clinico e componenti critici per apparecchiature come i ventilatori. II team CERN against COVID-19 si è subito attivato con il settore ricerca \& sviluppo per la progettazione e la costruzione di prototipi di nuovi ventilatori Hi-Tech. Questi progetti includono il Mechanical Ventilator Milano, il progetto MVM guidato dall'Istituto Nazionale di Fisica Nucleare (INFN) in Italia e che coinvolge fisici di tutto il mondo, il ventilatore della comunità di fisica ad alta energia, High Energy Ventilator (HEV), il cui sviluppo è guidato dalla collaborazione Large Hadron Collider beauty (LHCb), e un altro guidato dal laboratorio Laboratory of Instrumentation and Experimental Particle Physics (LIPP) in Portogallo, come Progetto Open Air. Questi progetti saranno pubblicati utilizzando la Licenza Open Hardware CERN, in modo che possano essere riprodotti ovunque sia necessario e adattati liberamente per conformarsi ai quadri normativi locali.

2) Informatica e analisi dei dati. II CERN è il centro hub di una vasta risorsa informatica globale, la Worldwide LHC Computing Grid (WLCG) ed è anche sede della collaborazione openlab del CERN con attori chiave nel settore dell'Information Technology. Ciò rappresenta una considerevole potenziale risorsa per combattere la pandemia, con potenziali applicazioni che vanno dal supporto della terapia e della ricerca sui vaccini all'implementazione della piattaforma di condivisione dei dati denominata Zenodo, che è un archivio open access per le pubblicazioni e l'archiviazione dei dati da parte dei ricercatori.

In tutti questi casi, il CERN è in stretto contatto con la comunità medica, attraverso, per esempio, l'accordo di collaborazione con l'Organizzazione Mondiale della Sanità.

Altri modi in cui vengono impiegate le risorse informatiche includono il supporto di un hackathon, che è un evento al quale partecipano, a vario titolo, esperti di diversi settori dell'informatica: sviluppatori di software, programmatori e grafici, e l'impiego di strumenti per l'apprendimento a distanza, come Open Up2U, con la partnership di ricerca e istruzione nazionale europea organizzazione di reti da parte del National Research and Education Network (NREN). II CERN sta contribuendo con risorse informatiche a un'iniziativa di informatica volontaria che mira a comprendere meglio il virus del COVID-19. L'iniziativa è in corso sulla piattaforma Folding@home, che è un progetto di calcolo distribuito per simulare la dinamica delle molecole proteiche. Come altri virus, il virus responsabile del COVID-19 ha proteine che vengono utilizzate per sopprimere il sistema immunitario degli ospiti, nonché proteine per l'auto-replicazione. Folding@ home sta supportando il lavoro per comprendere meglio queste proteine, che è un primo passo fondamentale verso lo sviluppo di nuovi farmaci che potrebbero inibire la loro funzione, arrestando, così, il virus. Mentre esistono molti metodi per determinare le strutture proteiche in laboratorio, questi approcci rivelano la forma in un dato momento. Le simulazioni al computer svolgono, quindi, un ruolo importante nel determinare come la struttura tridimensionale delle proteine possa cambiare nel tempo. Nel caso delle proteine virali, 
questo può rivelare nuovi siti in cui i farmaci possono essere utilizzati per attaccare la proteina e interromperne il funzionamento. Il numero di ricercatori in tutto il mondo che hanno contribuito a Folding@home è cresciuto rapidamente nelle ultime settimane. La massima potenza di calcolo collettiva del sistema è, ora, pari a quasi 2,5 exaFLOPS, che è superiore alla potenza di calcolo dei 500 supercomputer al mondo messi insieme. II CERN sta contribuendo con circa 10.000 core di computer dal suo principale data center. Tuttavia, ciò rappresenta solo circa un terzo delle "unità di lavoro" che il team del CERN ha completato per Folding@home. II resto proviene dai contributi forniti direttamente dai siti di elaborazione $\mathrm{LHCb}$.

Nella Tabella I sono mostrate le Aree d'azione del progetto “CERN Against COVID-19"

TABELLA I - Aree d'azione del progetto "CERN Against COVID-19"

\begin{tabular}{|c|c|}
\hline Area d'azione & Progetto \\
\hline Applicazioni mediche & Gel disinfettante \\
\hline Applicazioni mediche & $\begin{array}{l}\text { Stampanti 3D per la produzione di } \\
\text { dispositivi di protezione individuale, quali } \\
\text { maschere e componenti per adattare le } \\
\text { maschere disponibili in commercio per } \\
\text { uso clinico }\end{array}$ \\
\hline Applicazioni mediche & $\begin{array}{l}\text { Stampanti 3D per la produzione di } \\
\text { componenti critici per ventilatori }\end{array}$ \\
\hline Applicazioni mediche & Mechanical Ventilator Milano (MVM) \\
\hline Applicazioni mediche & High Energy Ventilator (HEV) \\
\hline $\begin{array}{l}\text { Informatica e analisi } \\
\text { dei dati }\end{array}$ & $\begin{array}{l}\text { Risorsa informatica globale Worldwide } \\
\text { LHC Computing Grid (WLCG) }\end{array}$ \\
\hline $\begin{array}{l}\text { Informatica e analisi } \\
\text { dei dati }\end{array}$ & $\begin{array}{l}\text { Piattaforma di condivisione dei dati } \\
\text { Zenodo }\end{array}$ \\
\hline $\begin{array}{l}\text { Informatica e analisi } \\
\text { dei dati }\end{array}$ & Condivisione dati Hackathon \\
\hline $\begin{array}{l}\text { Informatica e analisi } \\
\text { dei dati }\end{array}$ & Apprendimento a distanza Open Up2U \\
\hline $\begin{array}{l}\text { Informatica e analisi } \\
\text { dei dati }\end{array}$ & Piattaforma di calcolo Folding@home \\
\hline
\end{tabular}

\section{Ricerca farmacologica}

II primo studio randomizzato su malati COVID-19 nella ricerca farmacologica internazionale è un farmaco antivirale candidato, che è la combinazione degli inibitori delle proteasi nell'HIV lopinavir e ritonavir. II lopinavir presenta un'azione contro la protesasi $3 \mathrm{CL}$, nonché una modesta attività antivirale contro SARS-CoV-2 (7). La combinazione lopinavir e ritonavir incrementa la biodisponibilità del farmaco e, in questo momento, si sta studiando il loro effetto insieme all'immunomodulatore interferone beta- $1 \mathrm{~b}$ per il trattamento della sindrome respiratoria mediorientale (Middle East Respiratory Syndrome, MERS) in un trail clinico (ClinicalTrials.gov number, NCT02845843).
II 18 marzo 2020, The New England Journal of Medicine pubblica il primo RCT al mondo sugli effetti del trattamento con lopinavir-ritonavir nei pazienti con COVID-19 rispetto all'end-point primario il tempo del miglioramento clinico. Cao et al. (8), dal 18 gennaio 2020 al 3 febbraio 2020, al Jin Yin-Tan Hospital, Wuhan, Hubei Province, Cina, hanno condotto uno studio randomizzato controllato, open-label, in 199 pazienti adulti con diagnosi confermata in laboratorio di SARS-CoV-2. Di questi, 99 pazienti sono stati assegnati al gruppo in trattamento con lopinavir-ritonavir (rispettivamente $400 \mathrm{mg}$ e $100 \mathrm{mg}$ ) due volte al giorno, oralmente, per 14 giorni, e 100 pazienti sono stati assegnati al gruppo solo in standard care. A causa della necessità urgente di svolgere il trial, non è stato preparato il placebo lopinavir-ritonavir. I risultati hanno mostrato che il trattamento con lopinavir-ritonavir non era associato a differenze significative rispetto allo standard care sull'outcome miglioramento clinico (hazard ratio per il miglioramento clinico 1,$24 ; 95 \% \mathrm{Cl}, 0,90-1,72)$, mentre la mortalità a 28 giorni era similare nel gruppo lopinavir-ritonavir e nel gruppo in solo standard care $(19,2 \%$ vs $25,0 \%$; differenza, $-5,8$ punti percentuali; $95 \% \mathrm{Cl},-17,3-5,7)$. La percentuale di pazienti con RNA virale era similare nei vari time point tra $i$ due gruppi. Anche se il trattamento con lopinavir-ritonavir non ha mostrato benefici nei pazienti adulti con COVID-19, questo trial ha portato informazioni strategiche per stimolare la produzione di nuovi RCT di alta qualità da avviare rapidamente (9).

All'Agenzia Italiana del Farmaco è stato affidato il compito di valutare tutte le sperimentazioni cliniche sui medicinali per pazienti con COVID-19 (10).

In data 2 maggio sono 15 i trial clinici autorizzati dall'AIFA in riferimento ai farmaci tocilizumab, remdesivir, emapalumab, sarilumab, clorochina/idrossiclorochina, baricitinib, enoxaparina, colchicina, lopinavir con ritonavir e interferone (11).

Nella Tabella II sono mostrati i trial con i relativi outcome, popolazione di studio e tipo di intervento.

Nella Tabella III sono mostrati i trial con i relativi promotore, fase del trial, versione del protocollo e codici di registrazione.

\section{Farmaci per l'utilizzo contro COVID-19}

L'AIFA, al 3 maggio 2020, ha autorizzato I'utilizzo di vari farmaci contro COVID-19, I'utilizzo dell'azitromicina, da sola o associata ad altri farmaci, con particolare riferimento all'idrossoclorochina, al di fuori di eventuali sovrapposizioni batteriche, l'utilizzo del darunavir/cobicistat come inibitore delle proteasi, I'utilizzo di enoxaparina, I'unica eparina a basso peso molecolare (EBPM) ad avere l'indicazione nella profilassi del tromboembolismo venoso dei pazienti non chirurgici, il già citato lopinavir/ritonavir e l'idrossiclorochina (HCQ), che, insieme alla clorochina (CQ) (e ai suoi metaboliti attivi), ha dimostrato in vitro o in modelli animali di possedere un effetto antivirale attraverso l'alterazione (aumento) 


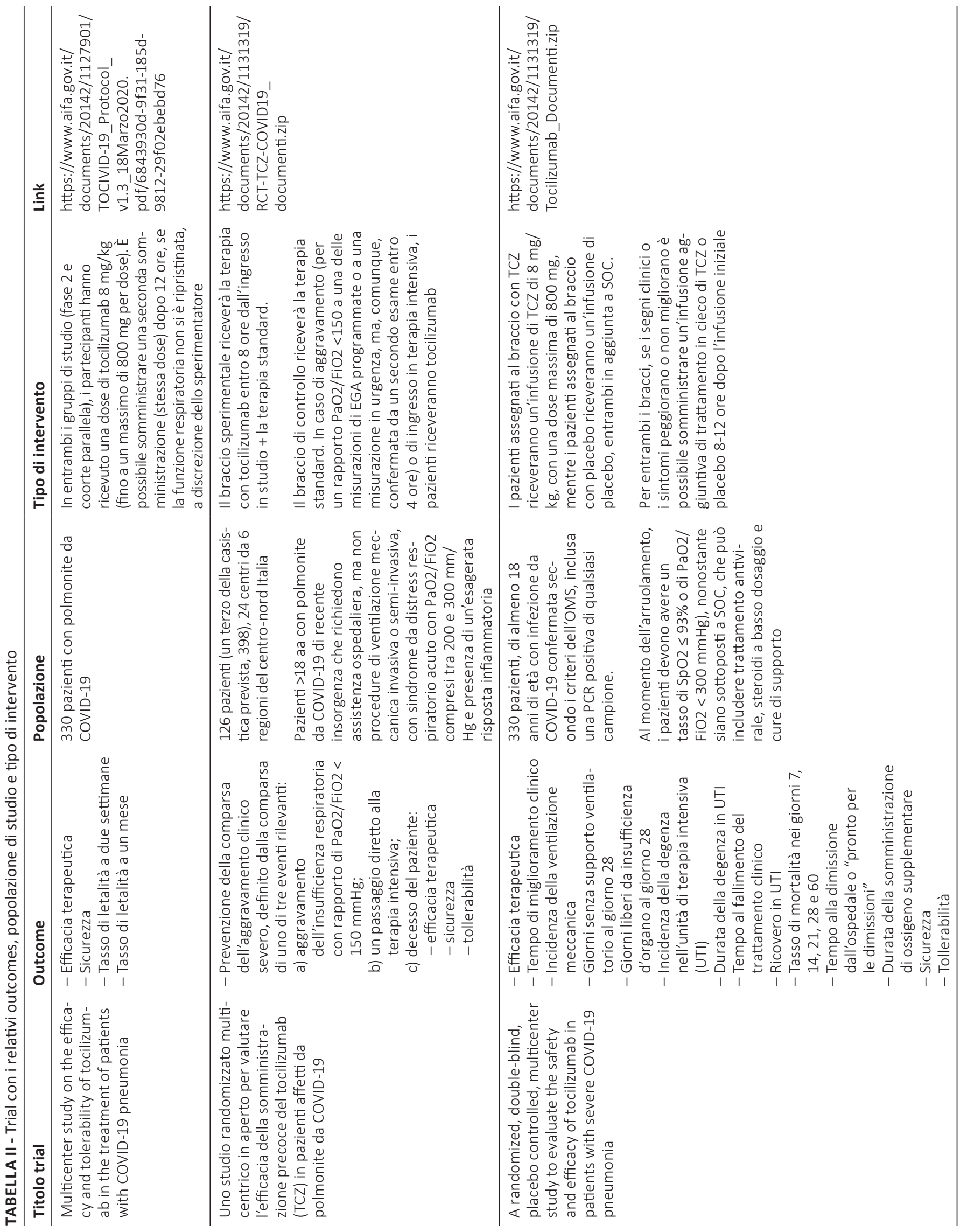




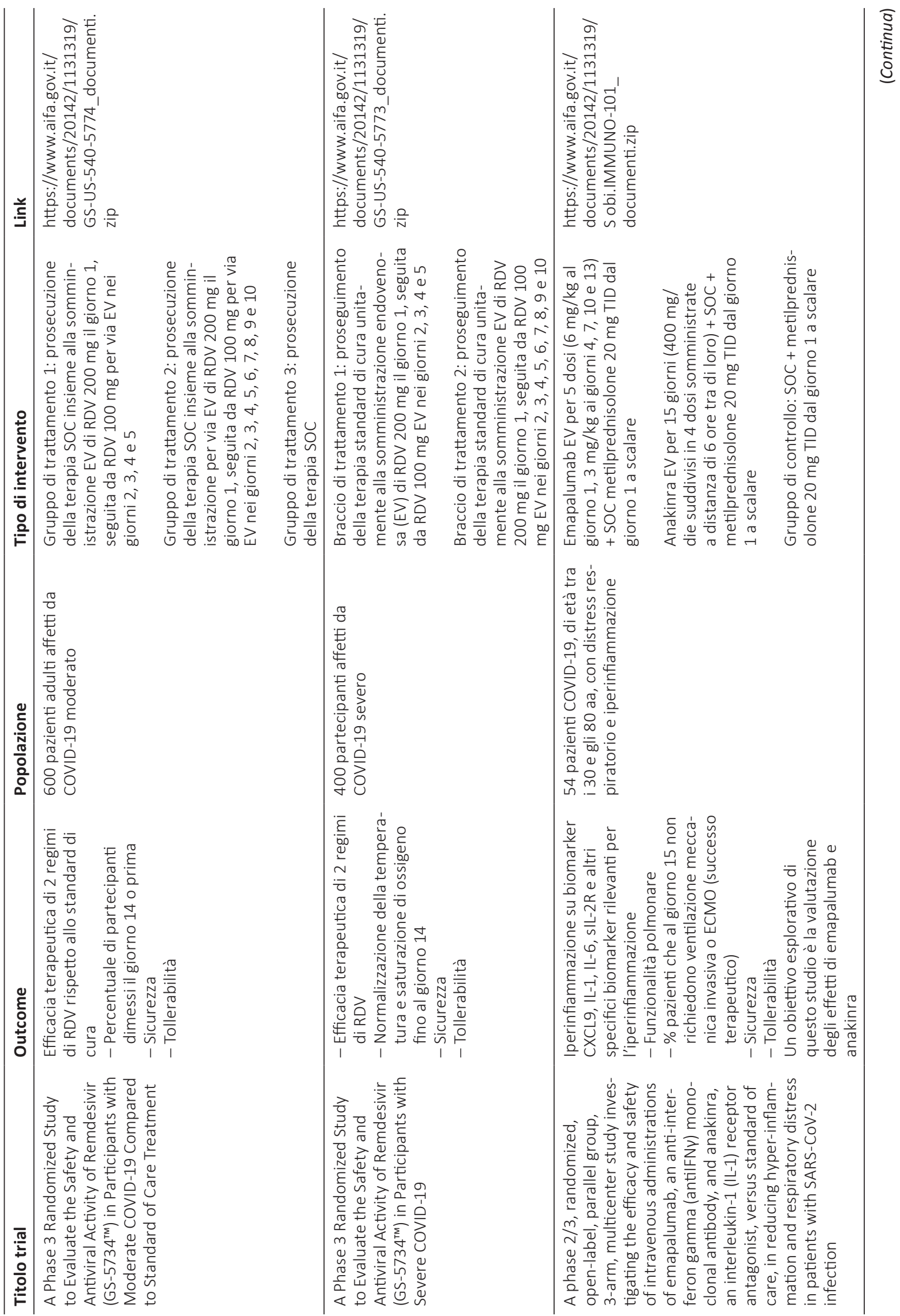


.

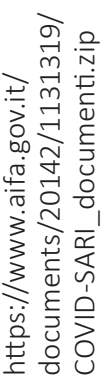

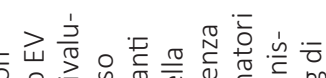

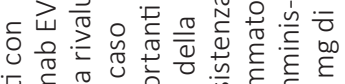

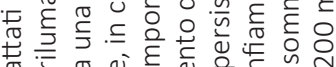

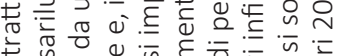

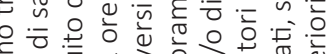

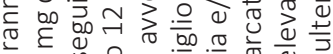

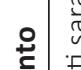

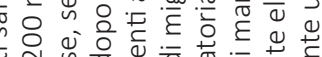

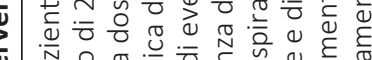

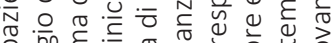

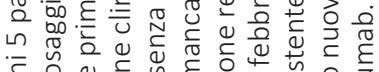

$\circ$ है

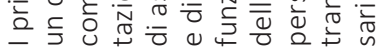

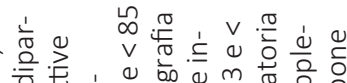

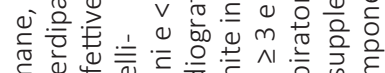

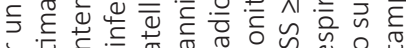
d

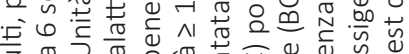
更西

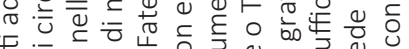

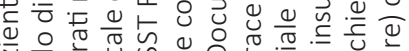

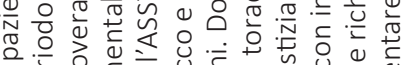

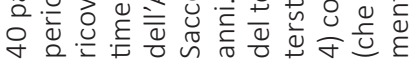

$$
\begin{aligned}
& \text { 结 究. }
\end{aligned}
$$

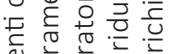

$$
\begin{aligned}
& \text { 은 흑 }
\end{aligned}
$$$$
\text { 중. }
$$

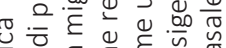

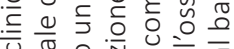

\section{$\stackrel{9}{\varepsilon}$} . $u$ 넌

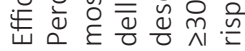

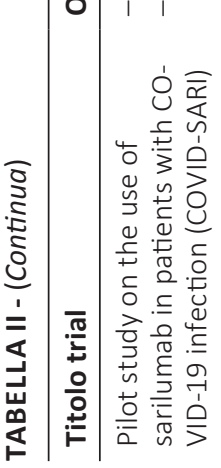

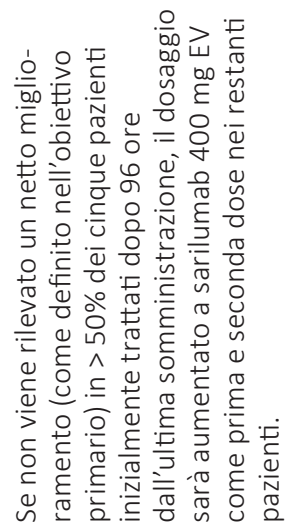

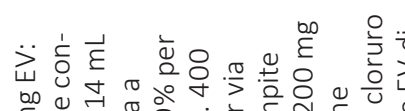

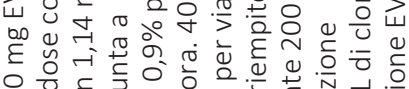

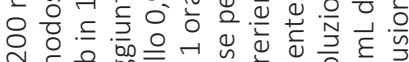
N

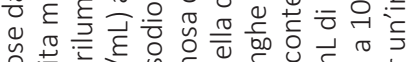

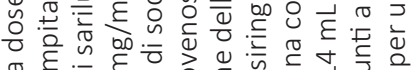

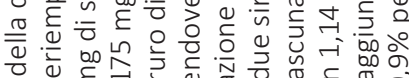

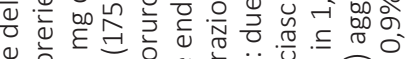

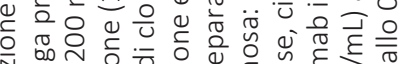

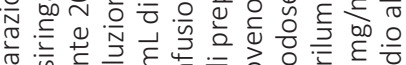

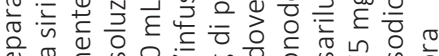

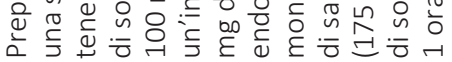

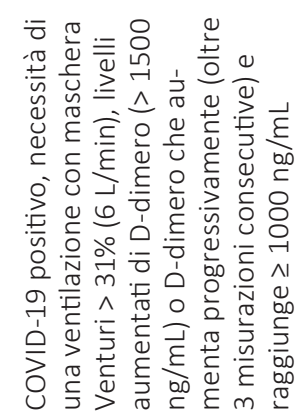

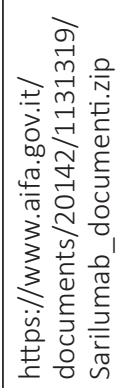

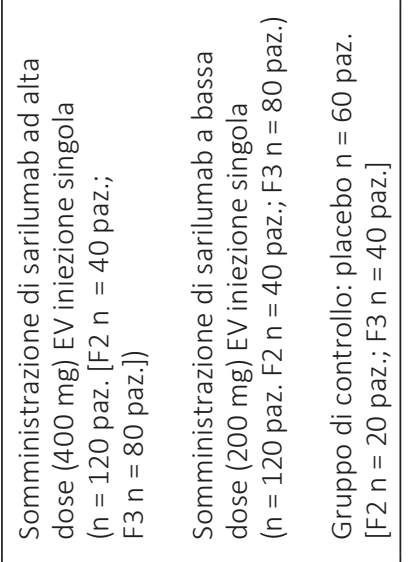
กั $\frac{1}{0} \frac{8}{0} \cdot \frac{1}{2}$ II ᄃ 11 过

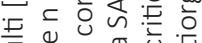
$\stackrel{2}{2}=\frac{\pi}{0}$

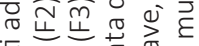

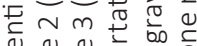

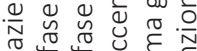

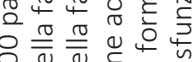

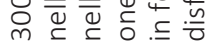

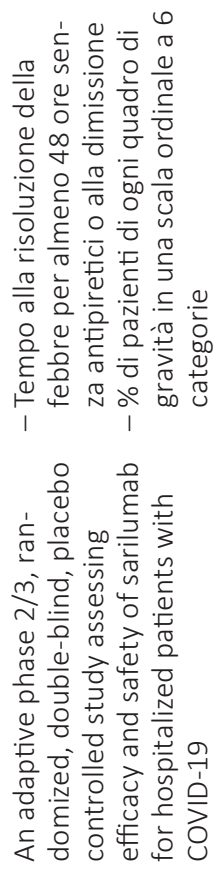




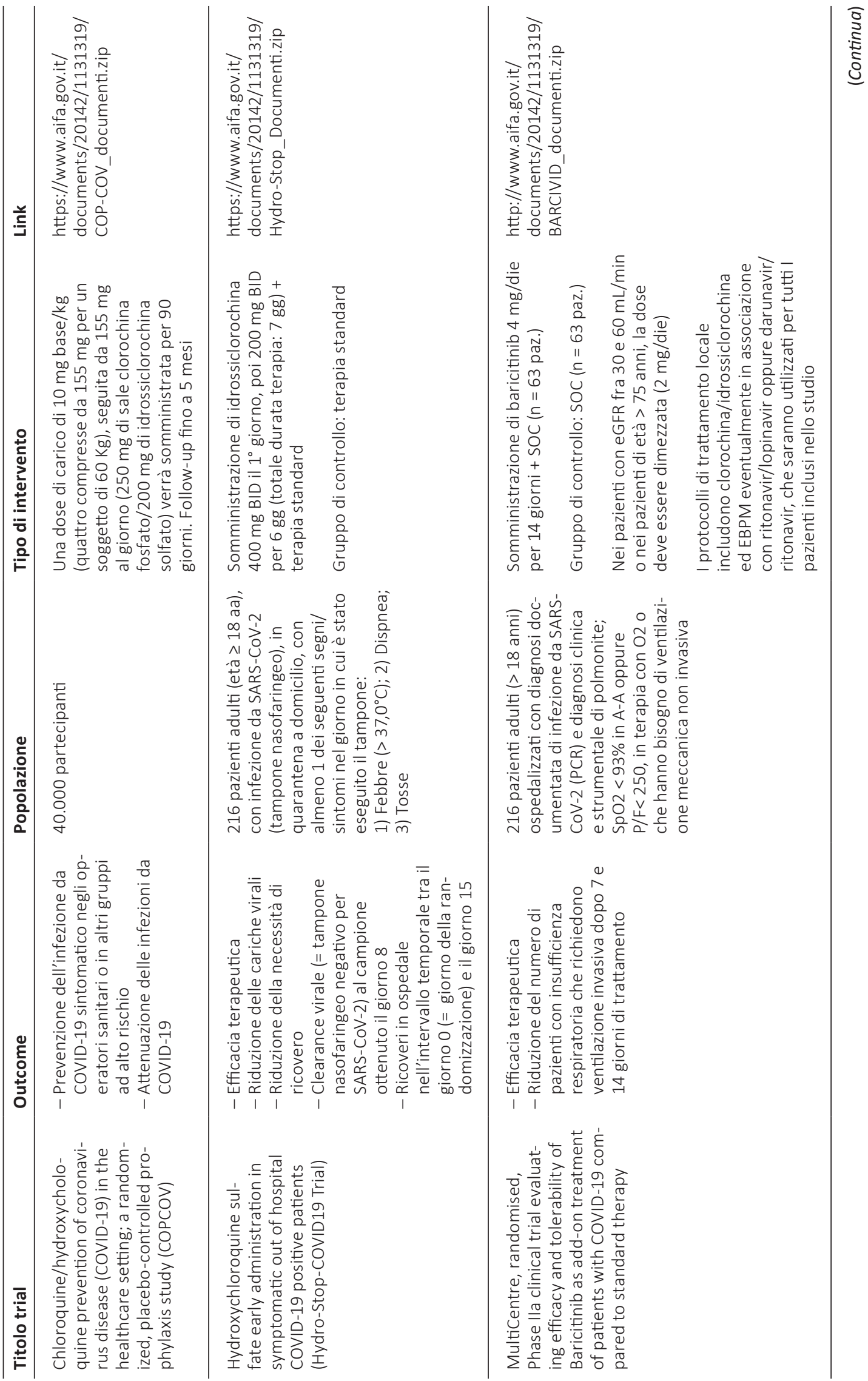




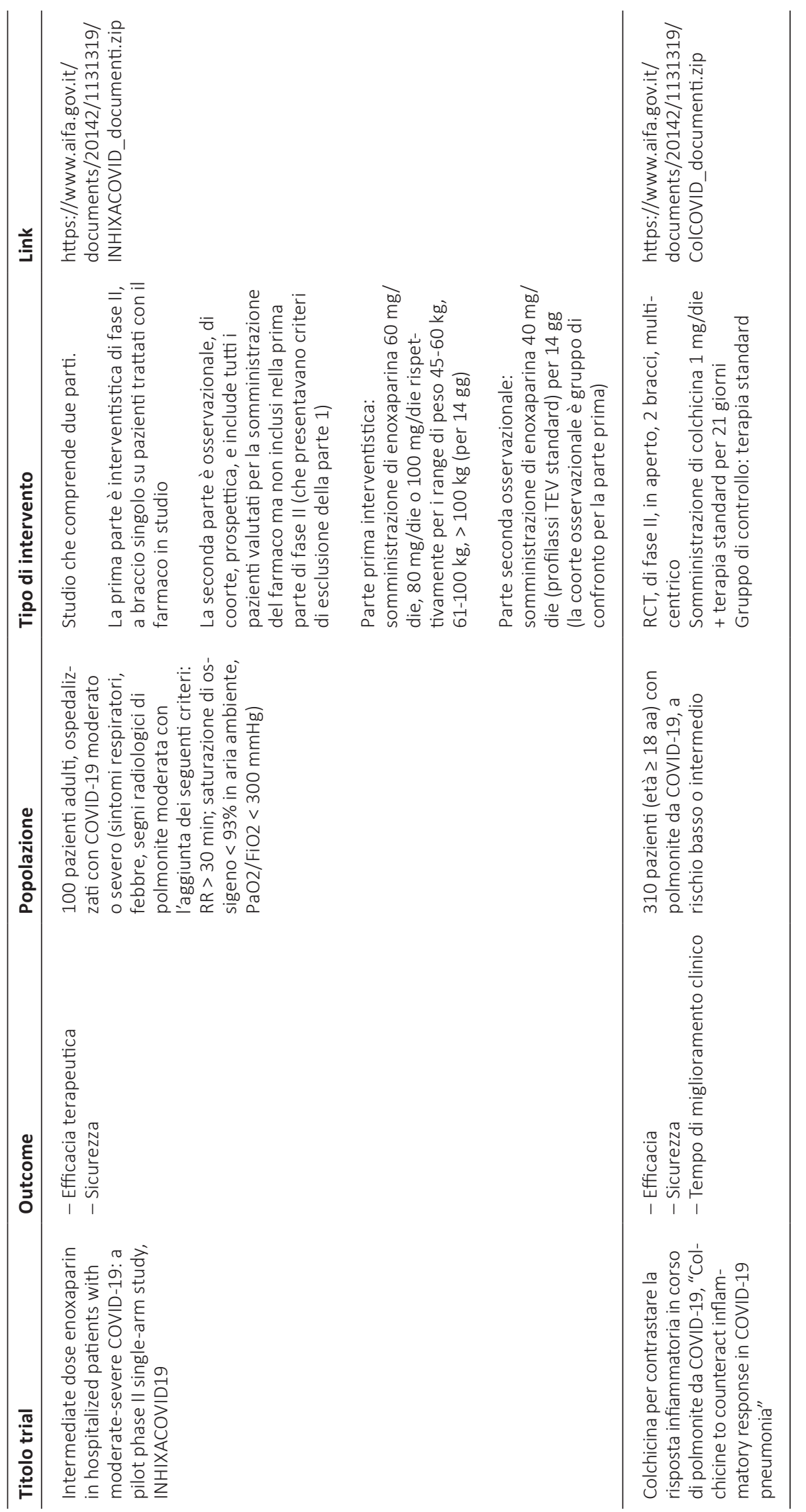




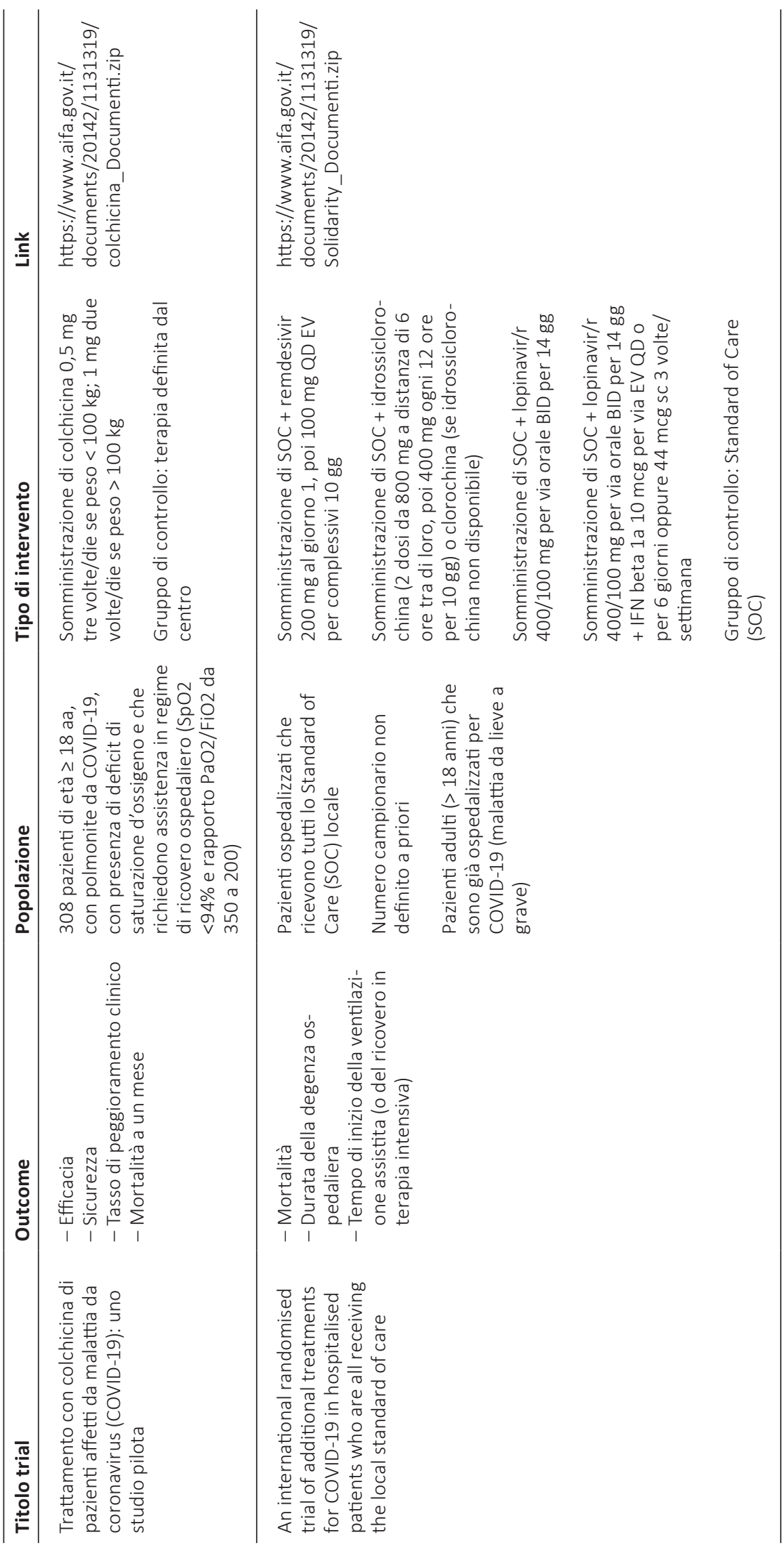


TABELLA III - Trial con i relativi promotore, fase del trial, versione del protocollo e codici di registrazione

\begin{tabular}{|c|c|c|c|c|}
\hline Titolo trial & Promotore & $\begin{array}{l}\text { Fase } \\
\text { del trial }\end{array}$ & $\begin{array}{l}\text { Versione } \\
\text { Protocollo }\end{array}$ & $\begin{array}{l}\text { Codici } \\
\text { Registrazione }\end{array}$ \\
\hline $\begin{array}{l}\text { Multicenter study on the efficacy and tolerability of } \\
\text { tocilizumab in the treatment of patients with COVID-19 } \\
\text { pneumonia }\end{array}$ & $\begin{array}{l}\text { Istituto Nazionale } \\
\text { Tumori }\end{array}$ & 2 & $\begin{array}{l}\text { n. } 1.3 \text { marzo } \\
18 \text { del } 2020\end{array}$ & $\begin{array}{l}\text { - EudraCT Number } \\
\text { 2020-001110-38 } \\
\text { - ClinicalTrials. } \\
\text { gov identifier } \\
\text { NCT04317092 }\end{array}$ \\
\hline $\begin{array}{l}\text { Uno studio randomizzato multicentrico in aperto per valutare } \\
\text { l'efficacia della somministrazione precoce del tocilizumab } \\
\text { (TCZ) in pazienti affetti da polmonite da COVID-19 }\end{array}$ & $\begin{array}{l}\text { Azienda Unità } \\
\text { Sanitaria Locale- } \\
\text { IRCCS di Reggio } \\
\text { Emilia }\end{array}$ & 2 & $\begin{array}{l}\text { n. } 2 \text { del } 25 \\
\text { marzo } 2020\end{array}$ & $\begin{array}{l}\text { EudraCT Number } \\
\text { 2020-001386-37 }\end{array}$ \\
\hline $\begin{array}{l}\text { A randomized, double-blind, placebo controlled, multicenter } \\
\text { study to evaluate the safety and efficacy of tocilizumab in } \\
\text { patients with severe COVID-19 pneumonia }\end{array}$ & $\begin{array}{l}\text { F. Hoffmann-La } \\
\text { Roche Ltd }\end{array}$ & 2 & $\begin{array}{l}\text { n. } 1 \text { del } 18 \\
\text { marzo } 2020\end{array}$ & $\begin{array}{l}\text { EudraCT Number } \\
\text { 2020-001154-22 }\end{array}$ \\
\hline $\begin{array}{l}\text { A Phase } 3 \text { Randomized Study to Evaluate the Safety and } \\
\left.\text { Antiviral Activity of Remdesivir (GS-5734 }{ }^{\mathrm{TM}}\right) \text { in Participants } \\
\text { with Moderate COVID-19 Compared to Standard of Care } \\
\text { Treatment }\end{array}$ & Gilead Sciences, Inc & 3 & $\begin{array}{l}\text { n. } 1 \text { del } 24 \\
\text { febbraio } 2020\end{array}$ & $\begin{array}{l}\text { - EudraCT Number } \\
\text { 2020-000842-32 } \\
\text { - ClinicalTrials. } \\
\text { gov identifier } \\
\text { NCT04292899 }\end{array}$ \\
\hline $\begin{array}{l}\text { A Phase } 3 \text { Randomized Study to Evaluate the Safety and } \\
\text { Antiviral Activity of Remdesivir (GS-5734 }{ }^{\mathrm{TM}} \text { ) in Participants } \\
\text { with Severe COVID-19 }\end{array}$ & Gilead Sciences, Inc & 3 & $\begin{array}{l}\text { n. } 1 \text { del } 24 \\
\text { febbraio } 2020\end{array}$ & $\begin{array}{l}\text { EudraCT Number } \\
\text { 2020-000841-15 } \\
\text { e ClinicalTrials. } \\
\text { gov identifier } \\
\text { NCT04292899 }\end{array}$ \\
\hline $\begin{array}{l}\text { A phase 2/3, randomized, open-label, parallel group, 3-arm, } \\
\text { multicenter study investigating the efficacy and safety of } \\
\text { intravenous administrations of emapalumab, an anti-interferon } \\
\text { gamma (antilFNy) monoclonal antibody, and anakinra, an } \\
\text { interleukin-1 (IL-1) receptor antagonist, versus standard of care, } \\
\text { in reducing hyper-inflammation and respiratory distress in } \\
\text { patients with SARS-CoV-2 infection }\end{array}$ & $\begin{array}{l}\text { Swedish Orphan } \\
\text { Biovitrum AB }\end{array}$ & $2-3$ & $\begin{array}{l}\text { n. } 3.0 \text { del } 20 \\
\text { marzo } 2020\end{array}$ & $\begin{array}{l}\text { EudraCT Number } \\
\text { 2020-001167-93 }\end{array}$ \\
\hline $\begin{array}{l}\text { Pilot study on the use of sarilumab in patients with COVID-19 } \\
\text { infection (COVID-SARI) }\end{array}$ & $\begin{array}{l}\text { ASST Fatebenefratelli } \\
\text { Sacco di Milano. }\end{array}$ & 2 & $\begin{array}{l}\text { n. } 3.1 \text { del } 15 \\
\text { aprile } 2020\end{array}$ & $\begin{array}{l}\text { EudraCT Number } \\
2020-001745-40\end{array}$ \\
\hline $\begin{array}{l}\text { An adaptive phase } 2 / 3 \text {, randomized, double-blind, placebo } \\
\text { controlled study assessing efficacy and safety of sarilumab for } \\
\text { hospitalized patients with COVID-19 }\end{array}$ & Sanofi- Aventis. & $2-3$ & & EFC16844 \\
\hline $\begin{array}{l}\text { Chloroquine/hydroxycholoquine prevention of coronavirus } \\
\text { disease (COVID-19) in the healthcare setting; a randomized, } \\
\text { placebo-controlled prophylaxis study (COPCOV) }\end{array}$ & Università di Oxford & 2 & $\begin{array}{l}\text { n. } 2 \text { del } 23 \\
\text { marzo } 2020\end{array}$ & $\begin{array}{l}\text { EudraCT Number } \\
\text { 2020-001441-39 }\end{array}$ \\
\hline $\begin{array}{l}\text { Hydroxychloroquine sulfate early administration in } \\
\text { symptomatic out of hospital COVID-19 positive patients } \\
\text { (Hydro-Stop-COVID-19 Trial) }\end{array}$ & $\begin{array}{l}\text { ASUR-AV5 Ascoli } \\
\text { Piceno }\end{array}$ & 2 & $\begin{array}{l}\text { n. } 1 \text { dell' } 8 \\
\text { aprile } 2020\end{array}$ & $\begin{array}{l}\text { EudraCT Number } \\
\text { 2020-001558-23 }\end{array}$ \\
\hline $\begin{array}{l}\text { MultiCentre, randomised, Phase lla clinical trial evaluating } \\
\text { efficacy and tolerability of Baricitinib as add-on treatment of } \\
\text { patients with COVID-19 compared to standard therapy }\end{array}$ & $\begin{array}{l}\text { Azienda Ospedaliera } \\
\text { Universitaria Pisana }\end{array}$ & 2 & $\begin{array}{l}\text { n. } 2.1 \text { del } 9 \\
\text { aprile } 2020\end{array}$ & $\begin{array}{l}\text { EudraCT Number } \\
\text { 2020-001955-42 }\end{array}$ \\
\hline $\begin{array}{l}\text { Intermediate dose enoxaparin in hospitalized patients with } \\
\text { moderate-severe COVID-19: a pilot phase II single-arm study, } \\
\text { INHIXACOVID19 }\end{array}$ & Università di Bologna & 2 & $\begin{array}{l}\text { n. } 1 \text { del } 18 \\
\text { marzo } 2020\end{array}$ & $\begin{array}{l}\text { EudraCT Number } \\
\text { 2020-001308-40 }\end{array}$ \\
\hline $\begin{array}{l}\text { Colchicina per contrastare la risposta infiammatoria in } \\
\text { corso di polmonite da COVID-19, "Colchicine to counteract } \\
\text { inflammatory response in COVID-19 pneumonia", con codice } \\
\text { di registrazione }\end{array}$ & $\begin{array}{l}\text { Azienda Ospedaliero- } \\
\text { Universitaria di } \\
\text { Parma }\end{array}$ & 2 & $\begin{array}{l}\text { n. } 1.1 \text { del } 14 \\
\text { aprile } 2020\end{array}$ & $\begin{array}{l}\text { EudraCT Number } \\
\text { 2020-001258-23 }\end{array}$ \\
\hline $\begin{array}{l}\text { Trattamento con colchicina di pazienti affetti da malattia da } \\
\text { coronavirus (COVID-19): uno studio pilota }\end{array}$ & $\begin{array}{l}\text { Azienda Ospedaliera } \\
\text { di Perugia }\end{array}$ & 2 & $\begin{array}{l}\text { n. } 1.13 \text { del } 6 \\
\text { aprile } 2020\end{array}$ & $\begin{array}{l}\text { EudraCT Number } \\
\text { 2020-001475-33 }\end{array}$ \\
\hline $\begin{array}{l}\text { An international randomised trial of additional treatments for } \\
\text { COVID-19 in hospitalised patients who are all receiving the } \\
\text { local standard of care }\end{array}$ & Università di Verona & 2 & $\begin{array}{l}\text { n. } 10 \text { del } 22 \\
\text { marzo } 2020\end{array}$ & $\begin{array}{l}\text { EudraCT Number } \\
\text { 2020-001366-11 }\end{array}$ \\
\hline
\end{tabular}


del $\mathrm{pH}$ endosomiale, che è determinante per la fusione viruscellula. Tali farmaci, inoltre, interferiscono sulla glicosilazione dei recettori cellulari di SARS-CoV-2.

\section{Farmaci ad uso compassionevole}

Gli attuali farmaci autorizzati dall'AlFA per un utilizzo compassionevole nel trattamento dei pazienti con COVID-19 sono: canakinumab (Ilaris), remdesivir, ruxolitinib, solnatide.

Nella Tabella IV sono mostrati i trial con i relativi outcome, popolazione di studio, tipo di intervento e link.

Nella Tabella $\mathrm{V}$ sono mostrati i trial con i relativi promotore, versione del protocollo e codice Registro delle sperimentazioni.

\section{Analisi wavelet e modellistica deterministica}

Krantz e Srinivasa Rao (12) propongono una valutazione basata su modelli di sottostima del coronavirus (COVID-19) in vari paesi in tutto il mondo, usando i metodi recentemente sviluppati con I'analisi armonica, per esempio sviluppando dati epidemici completi partendo da dati parziali usando l'approccio wavelet. Un modello permette di portare nuove informazioni, soprattutto predittive su ciò che non è stato direttamente osservato. Questo modello permette di predire variabili, come, per esempio, la persistenza del virus, o di calcolare la probabilità di trasmissione, dati importanti per la programmazione sanitaria. I ricercatori hanno raccolto dati sul COVID-19 dalle fonti ufficiali della WHO e del

TABELLA IV - Trial con i relativi outcome, popolazione di studio, tipo di intervento e link

\begin{tabular}{|c|c|c|c|c|}
\hline Titolo di trial & Outcome & Popolazione & Tipo di intervento & Link \\
\hline $\begin{array}{l}\text { Managed Access } \\
\text { Program (MAP) to } \\
\text { provide access to } \\
\text { canakinumab (Ilaris) } \\
\text { treatment of cytokine } \\
\text { release syndrome } \\
\text { (CRS) in patients with } \\
\text { COVID-19-induced } \\
\text { pneumonia }\end{array}$ & $\begin{array}{l}\text { - Profilazione delle } \\
\text { citochine nei pazienti } \\
\text { IL-1, IL-2, IL-7, IL-6 } \\
\text { - Stimolazione delle } \\
\text { colonie di granulociti } \\
\text { - Interferone- }-10 \\
\text { - Proteina infiammatoria } \\
\text { macrofagi 1- } \alpha \\
\text { - Fattore di necrosi } \\
\text { tumorale- } \alpha\end{array}$ & $\begin{array}{l}\text { Età } \geq 18 \text { anni } \\
\text { Diagnosi clinica del virus } \\
\text { SARS-CoV-2 mediante } \\
\text { PCR o mediante altra } \\
\text { metodologia diagnostica } \\
\text { approvata o, con } \\
\text { diagnosi presuntiva di } \\
\text { COVID-19 }\end{array}$ & $\begin{array}{l}\text { La dose di canakinumab } \\
\text { è di } 600 \mathrm{mg} \text { in } 250 \mathrm{~mL} \text { di } \\
\text { destrosio al } 5 \% \text { infuso per } \\
\text { via endovenosa per } 2 \text { ore } \\
\text { La dose non deve } \\
\text { superare circa } 10 \mathrm{mg} / \mathrm{kg}\end{array}$ & $\begin{array}{l}\text { https://www.aifa.gov.it/ } \\
\text { documents/20142/1143282/ } \\
\text { Canakinumab_documenti.zip }\end{array}$ \\
\hline $\begin{array}{l}\text { Expanded Access } \\
\text { Treatment Protocol: } \\
\text { Remdesivir (RDV; } \\
\text { GS-5734) for the } \\
\text { Treatment of SARS- } \\
\text { CoV-2 (CoV) Infection }\end{array}$ & $\begin{array}{l}\text { - Tasso di incidenza } \\
\text { di eventi avversi } \\
\text { emergenti dal } \\
\text { trattamento }\end{array}$ & $\begin{array}{l}5000 \text { partecipanti di } \\
\text { età } \geq 18 \text { anni, ricoverati } \\
\text { con SARS-CoV-2 } \\
\text { confermato mediante } \\
\text { PCR o contatto noto del } \\
\text { caso confermato con } \\
\text { sindrome coerente con } \\
\text { COVID-19 con PCR e } \\
\text { richiesta di ventilazione } \\
\text { meccanica invasiva con } \\
\text { funzione renale adeguata } \\
\text { con velocità di filtrazione } \\
\text { glomerulare stimata } \geq 30 \\
\text { mL/min e ALT } \leq 5 \times \text { limite } \\
\text { superiore della norma }\end{array}$ & $\begin{array}{l}\text { Dose singola di carico per } \\
\text { RDV } 200 \text { mg EV il giorno } \\
1 \text { di trattamento seguito } \\
\text { da dosi di mantenimento } \\
\text { giornaliere di } 100 \text { mg EV } \\
\text { nei giorni } 2-10 \text {, per un } \\
\text { massimo di } 10 \text { giorni in } \\
\text { totale }\end{array}$ & $\begin{array}{l}\text { https://www.aifa.gov.it/ } \\
\text { documents/20142/1144520/ } \\
\text { Remdesivir_documenti.zip }\end{array}$ \\
\hline $\begin{array}{l}\text { Ruxolitinib Managed } \\
\text { Access Program } \\
\text { (MAP) for patients } \\
\text { diagnosed with } \\
\text { COVID-19 and have } \\
\text { severe/very severe } \\
\text { lung disease (CINC424, } \\
\text { RUXOLITINIB, JAKAVI) }\end{array}$ & $\begin{array}{l}\text { Accesso alla terapia con } \\
\text { ruxolitinib per i pazienti } \\
\text { eleggibili con diagnosi di } \\
\text { malattia COVID-19 grave/ } \\
\text { molto grave }\end{array}$ & $\begin{array}{l}\text { 1. Pazienti di età } \geq 6 \text { anni } \\
\text { 2. Pazienti con diagnosi } \\
\text { clinica di infezione } \\
\text { da SARS-CoV-2, } \\
\text { attraverso anticorpi } \\
\text { sierici positivi (IgM } \\
\text { o IgG) o mediante } \\
\text { PCR o mediante } \\
\text { altra metodologia } \\
\text { diagnostica approvata }\end{array}$ & $\begin{array}{l}\text { Per pazienti di età } \geq 6-<12 \\
\text { anni, la dose è di } 5 \text { mg QD } \\
\text { (una volta al giorno) } \\
\text { Per pazienti di età } \geq 12 \\
\text { anni, la dose è di } 5 \text { mg BID } \\
\text { La durata raccomandata } \\
\text { del trattamento è di } 7 \\
\text { giorni, seguita da una } \\
\text { valutazione clinica/ } \\
\text { radiologica. II trattamento } \\
\text { può essere prolungato } \\
\text { fino a } 28 \text { giorni, se } \\
\text { clinicamente indicato, e i } \\
\text { benefici del trattamento } \\
\text { superano i rischi }\end{array}$ & $\begin{array}{l}\text { https://www.aifa.gov.it/ } \\
\text { documents/20142/1140695/ } \\
\text { Ruxolitinib_documenti.zip }\end{array}$ \\
\hline
\end{tabular}


TABELLA IV - (Continua)

\begin{tabular}{|c|c|c|c|c|}
\hline Titolo di trial & Outcome & Popolazione & Tipo di intervento & Link \\
\hline $\begin{array}{l}\text { Programma di Uso } \\
\text { Compassionevole } \\
\text { con solnatide per } \\
\text { il trattamento } \\
\text { dell'edema da } \\
\text { permeabilità } \\
\text { polmonare in pazienti } \\
\text { affetti da COVID-19 } \\
\text { con insufficienza } \\
\text { polmonare acuta }\end{array}$ & $\begin{array}{l}\text { Edema da permeabilità } \\
\text { polmonare }\end{array}$ & $\begin{array}{l}20 \text { pazienti adulti } \\
\text { positivi per SARS- } \\
\text { CoV-2 e ricoverati } \\
\text { in ICU e ventilati } \\
\text { meccanicamente } \\
\text { Presenza di ARDS con } \\
\text { insorgenza acuta di } \\
\text { insufficienza respiratoria } \\
\text { ipossiemica, infiltrati } \\
\text { bilaterali, PaO2/FiO2 } \\
\leq 300 \text { mmHg e pressione } \\
\text { positiva di fine } \\
\text { espirazione (Positive } \\
\text { End-Expiratory Pressure, } \\
\text { PEEP) } \geq 5 \text { cm H2O }\end{array}$ & $\begin{array}{l}\text { Somministrazione per via } \\
\text { inalatoria endotracheale, } \\
\text { tramite dispositivo } \\
\text { nebulizzatore (Aeroneb }{ }^{\circledR} \text { ), } \\
\text { di } 100 \text { mg due volte al } \\
\text { giorno per massimo } 7 \\
\text { giorni }\end{array}$ & $\begin{array}{l}\text { https://www.aifa.gov.it/ } \\
\text { documents/20142/1131319/ } \\
\text { Solnatide_documenti.zip }\end{array}$ \\
\hline
\end{tabular}

TABELLA V - Trial con i relativi promotore, versione del protocollo e codice Registro delle sperimentazioni

\begin{tabular}{|c|c|c|c|}
\hline Titolo trial & Promotore & $\begin{array}{l}\text { Versione } \\
\text { Protocollo }\end{array}$ & $\begin{array}{l}\text { Registro delle } \\
\text { Sperimentazioni }\end{array}$ \\
\hline $\begin{array}{l}\text { Managed Access Program (MAP) to provide access to } \\
\text { canakinumab (Ilaris) treatment of cytokine release syndrome } \\
\text { (CRS) in patients with COVID-19-induced pneumonia }\end{array}$ & Novartis & 30 marzo 2020 & $\begin{array}{l}\text { n. } 57 \text { il } 07 / 04 / 2020 \\
\text { Comitato etico dell'INMI } \\
\text { Lazzaro Spallanzani di Roma }\end{array}$ \\
\hline $\begin{array}{l}\text { Expanded Access Treatment Protocol: Remdesivir } \\
\text { (RDV; GS-5734) for the Treatment of SARS-CoV-2 (CoV) } \\
\text { Infection }\end{array}$ & $\begin{array}{l}\text { Gilead Sciences, } \\
\text { Inc }\end{array}$ & $\begin{array}{l}\text { GS-US-540-5821 } \\
22 \text { marzo } 2020\end{array}$ & $\begin{array}{l}\text { n. } 34 \text { il 26/03/2020 } \\
\text { Comitato etico dell'INMI } \\
\text { Lazzaro Spallanzani di Roma }\end{array}$ \\
\hline $\begin{array}{l}\text { Ruxolitinib Managed Access Program (MAP) for patients } \\
\text { diagnosed with COVID-19 and have severe/very severe lung } \\
\text { disease (CINC424, RUXOLITINIB, JAKAVI) }\end{array}$ & Novartis & 25 marzo 2020 & $\begin{array}{l}\text { n. } 47 \text { il 02/04/2020 } \\
\text { Comitato etico dell'INMI } \\
\text { Lazzaro Spallanzani di Roma }\end{array}$ \\
\hline $\begin{array}{l}\text { Programma di Uso Compassionevole con solnatide per il } \\
\text { trattamento dell'edema da permeabilità polmonare in pazienti } \\
\text { affetti da COVID-19 con insufficienza polmonare acuta }\end{array}$ & $\begin{array}{l}\text { APEPTICO } \\
\text { Forschung und } \\
\text { Entwicklung } \mathrm{GmbH}\end{array}$ & $\begin{array}{l}1.0 \text { del } \\
14 \text { aprile } 2020\end{array}$ & $\begin{array}{l}\text { n. } 71 \text { il 15/04/2020 } \\
\text { Comitato etico dell'INMI } \\
\text { Lazzaro Spallanzani di Roma }\end{array}$ \\
\hline
\end{tabular}

Worldometer. Le variabili utilizzate sono la densità di popolazione, la percentuale della popolazione che vive nelle aree urbane, le popolazioni stratificate in tre fasce d'età, 0-14, $15-64,>65$, i nuovi casi giornalieri (>10), il primo picco di infezione e gli intervalli temporali, che variavano in un range tra $8 \mathrm{e}$ 16 giorni.

I ricercatori hanno utilizzato due equazioni differenziali accoppiate $s(t)=-\beta s(t) k(t)$ e $k(t) \beta s t(k) t$, dove $s(t)$ e $k(t)$ rappresentano i suscettibili e infetti al momento t e $\beta$ è la percentuale di trasmissione che si considera che sia invariante entro I'intervallo di giorni per il quale sono stati calcolati i numeri di infezioni in ciascun paese. La differenza tra i numeri previsti dal modello e i numeri effettivi riportati all'interno dell'intervallo sono stati trattati come sottostimati (compresi quelli sottodiagnosticati). Per esempio, in Italia, in base ai dati della WHO, il modello matematico indicava, al 9 marzo, 30.223 casi che non sono stati registrati.
Nella Tabella VI sono mostrati i casi, i dati demografici, i casi giornalieri, i tassi di crescita e le sottostime fino al 9 marzo 2020 (12).

\section{Conclusioni}

La pandemia causata dal SARS-CoV-2 virus ha potenziato enormemente le sinergie tra le varie modalità di ricerca \& sviluppo a livello internazionale. La possibilità di creare una modellistica genetica ed epidemiologica attraverso l'utilizzo della più grande potenza di calcolo e della più innovativa tecnologia a disposizione dell'uomo pone basi solide per una possibilità in tempi brevi di individuare un farmaco efficace e sicuro e di arrivare a una conoscenza precisa del virus e del suo comportamento. In una seconda parte di questo articolo, affronteremo i risultati degli studi farmacologici rispetto alle singole molecole sottoposte a sperimentazione. 
TABELLA VI - Casi, dati demografici, casi giornalieri, tassi di crescita e sottostime fino al 9 Marzo 2020

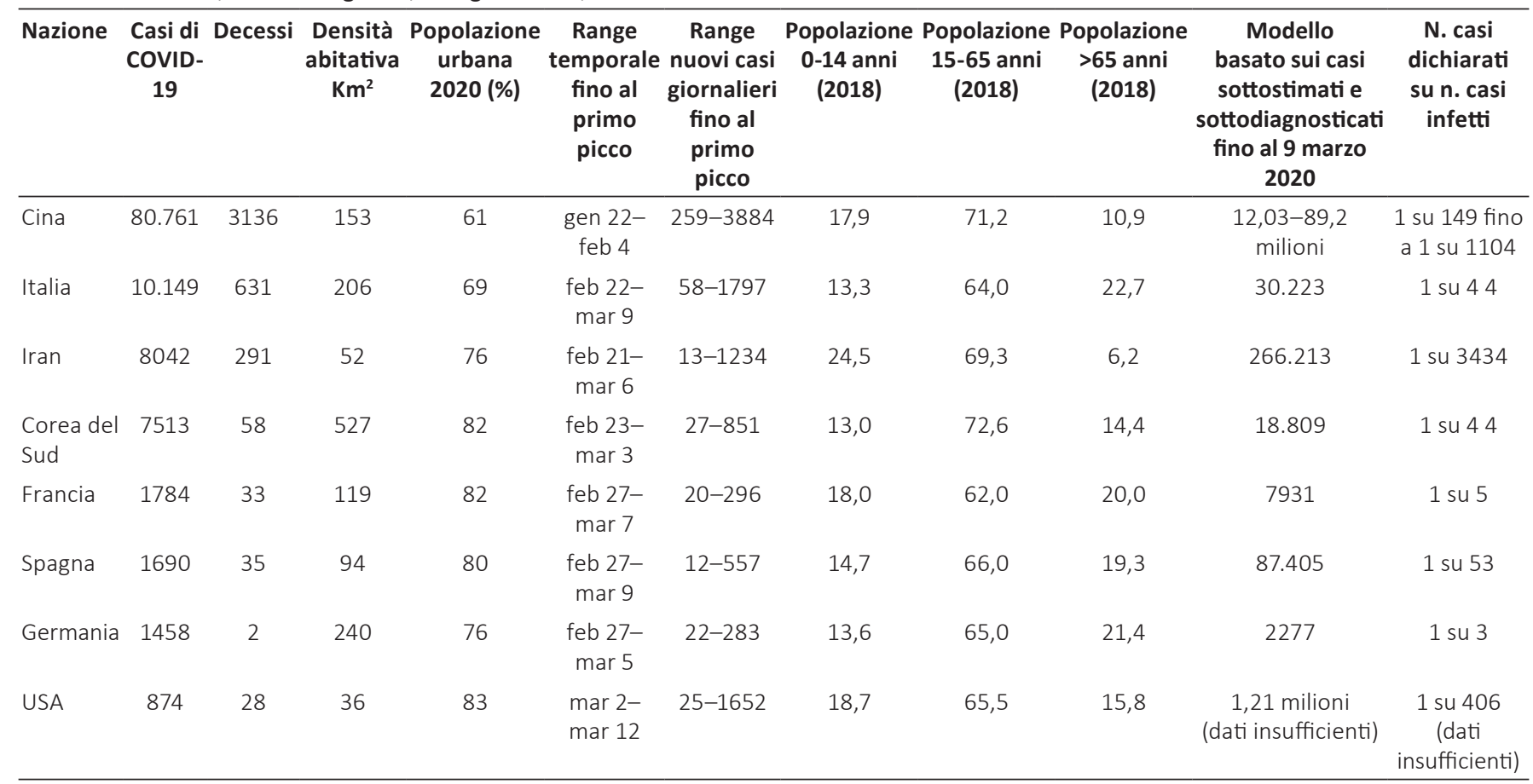

\section{Disclosures}

Conflict of interest: The authors declare no conflict of interest. Financial support: This research received no specific grant from any funding agency in the public, commercial, or not-for-profit sectors.

\section{Bibliografia}

1. Center for Disease Control and Prevention. Situation Summary. Centre for Disease Control and Prevention. 2020. https://www. cdc.gov/coronavirus/2019-ncov/cases updates/summary.html (Data ultimo accesso: 2/3/2020).

2. World Health Organization. WHO Director-General's opening remarks at the media briefing on COVID-19. 11 march 2020. https://www.who.int/dg/speeches/detail/who-director-general-sopening-remarks-at-the-media-briefing-on-covid-19---11-march2020 (Data ultimo accesso: 29/3 2020).

3. World Health Organization. Coronavirus disease 2019 (COVID-19) Situation Report - 67. 2020. https://www.who.int/ docs/default-source/coronaviruse/situation-reports/20200405sitrep-76-covid-19.pdf?sfvrsn=6ecf0977_4 (Data ultimo accesso: 2/5/2020).

4. World Health Organization. Global surveillance for COVID-19 caused by human infection with COVID-19 virus. 2020. https:// apps.who.int/iris/handle/10665/331506 (Data ultimo accesso: 20/3/2020).
5. Ministero della Salute. Circolare n. 6360 del 27/02/2020 "COVID-19". Aggiornamento.

6. National Health Institute. NIH clinical trial of remdesivir to treat COVID-19 begins. 2020. https://www.nih.gov/newsevents/news-releases/nih-clinical-trial-remdesivir-treat-covid19-begins (Data ultimo accesso: 29/3/2020).

7. Sheahan TP, Sims AC, Leist SR, et al. Comparative therapeutic efficacy of remdesivir and combination lopinavir, ritonavir, and interferon beta against MERS-CoV. Nat Commun. 2020;11:222. https://experience.arcgis.com/experience/685d0ace521648f8 a5beeee1b9125cd (Data ultimo accesso: 2/5/2020).

8. Cao B, Wang Y, Wen D, et al. A Trial of Lopinavir Ritonavir in Adults Hospitalized with Severe Covid-19. N Engl J Med. 2020; 382:1787-99. doi: 10.1056/NEJMoa2001282.

9. Baden LR, Rubin EJ. Covid-19-The Search for Effective Therapy. N Engl J Med. 2020; 382:1851-2. doi: 10.1056/NEJMe2005477.

10. Decreto Legge 17 marzo 2020, n. 18. https://www.gazzettaufficiale.it/eli/id/2020/03/17/20G00034/sg.

11. Agenzia Italiana del Farmaco. Sperimentazioni cliniche COVID-19. Agenzia Italiana del Farmaco. 2020. https://www. aifa.gov.it/sperimentazioni-cliniche-covid-19 (Data ultimo accesso: 2/5/2020).

12. Krantz SG, Srinivasa Rao ASR. Level of underreporting including underdiagnosis before the first peak of COVID-19 in various countries: Preliminary retrospective results based on wavelets and deterministic modeling. Infection Control \& Hospital Epidemiology. 2020;41:857-9. doi: 10.1017/ice.2020.116. 